\title{
Oscillation of second-order nonlinear difference equations with sublinear neutral term
}

\author{
Martin Bohner, Hassan A. El-Morshedy, \\ SAID R. Grace, Ilgin SAĞER
}

\begin{abstract}
We establish some new criteria for the oscillation of secondorder nonlinear difference equations with a sublinear neutral term. This is accomplished by reducing the involved nonlinear equation to a linear inequality.
\end{abstract}

\section{INTRODUCTION}

This paper deals with oscillatory behavior of all solutions of nonlinear second-order difference equations with a sublinear neutral term of the form

$$
\Delta\left(a_{n} \Delta\left(x_{n}+p_{n} x_{n-k}^{\alpha}\right)\right)+q_{n} x_{n+1-m}^{\beta}=0 .
$$

We assume that

$\left(\mathrm{H}_{1}\right) 0<\alpha<1$ and $\beta>0$ are ratios of positive odd integers,

$\left(\mathrm{H}_{2}\right)\left\{a_{n}\right\},\left\{p_{n}\right\},\left\{q_{n}\right\}, n \geq n_{0}$, are positive real sequences,

$$
\lim _{n \rightarrow \infty} p_{n}=0 \text { and } \sum_{s=n_{0}}^{\infty} \frac{1}{a_{s}}<\infty,
$$

$\left(\mathrm{H}_{3}\right) k \in \mathbb{N}$ and $m \in \mathbb{N}_{0}$.

Let $\xi=\max \{k, m-1\}$. By a solution of (1), we mean a real sequence $\left\{x_{n}\right\}$ defined for all $n \geq n_{0}-\xi$ that satisfies (1) for $n \geq n_{0}$. A solution of (1) is said to be oscillatory if its terms are neither eventually positive nor eventually negative, and otherwise it is called nonoscillatory. Equation (1) is said to be oscillatory if all its solutions are oscillatory.

In recent years, there has been a great interest in establishing criteria for the oscillation and asymptotic behavior of solutions of various classes of second-order difference equations, see $[1,2,4,9-12,15,18,20,21,24]$ and the references cited therein. However, it seems that there are no known results regarding the oscillation of second-order difference equations with positive

2010 Mathematics Subject Classification. Primary: 39A10, 39A21.

Key words and phrases. Neutral term, nonlinear difference equation, oscillation.

Full paper. Received 28 November 2018, accepted 28 December 2019, available online 17 January 2019. 
sublinear neutral term. More exactly, the existing literature does not provide any criteria which ensure oscillation of all solutions of (1). In view of this motivation, our aim in this paper is to present sufficient conditions which ensure that all solutions of (1) are oscillatory. For related results concerning second-order differential equations with sublinear neutral term, we refer the reader to $[3,16,17,23]$. Some related results concering second-order dynamic equations on time scales can be found in [6-8, 13, 14, 19, 22].

\section{Main Results}

For $n \geq n_{0}^{*}$ for some $n_{0}^{*} \geq n_{0}$, we let

$$
A_{n}=\sum_{s=n}^{\infty} \frac{1}{a_{s}} .
$$

For convenience, for some $0<\nu \leq 1$ and $n \geq n_{0}^{*}$, we set

$$
\begin{gathered}
y_{n}=x_{n}+p_{n} x_{n-k}^{\alpha}, \\
P_{n}=1-p_{n} \frac{A_{n-k}^{\alpha}}{A_{n}^{1+(1-\alpha) \nu}} \geq 0
\end{gathered}
$$

and

$$
Q_{n}=q_{n} A_{n+1}^{(1+\nu)(\beta-1)} P_{n+1-m}^{\beta} .
$$

In the following, we establish a new oscillation result for (1) when $\beta \geq 1$.

Theorem 2.1. Let $\beta \geq 1$. Assume $\left(H_{1}\right)-\left(H_{3}\right)$. If

$$
\limsup _{n \rightarrow \infty} \sum_{s=n_{0}^{*}}^{n}\left[Q_{s} A_{s+1}-\frac{1}{4 a_{s} A_{s+1}}\right]=\infty,
$$

then (1) is oscillatory.

Proof. Let $x_{n}$ be a nonoscillatory solution of (1), say $x_{n}>0, x_{n+1-m}>0$, $x_{n-k}>0$, and $y_{n}>0$ for $n \geq n_{1}$ for some $n_{1} \geq n_{0}^{*}$. It is easy to see that $y_{n}>0, n \geq n_{1}$, and (1) becomes

$$
\Delta\left(a_{n} \Delta y_{n}\right)+q_{n} x_{n+1-m}^{\beta}=0 .
$$

Thus $\Delta\left(a_{n} \Delta y_{n}\right) \leq 0$ for $n \geq n_{1}$, which implies that $y_{n}$ is bounded. Also, the decreasing nature of $a_{n} \Delta y_{n}$ implies that (I) $\Delta y_{n}>0$ or (II) $\Delta y_{n}<0$ for $n \geq n_{1}^{*} \geq n_{1}$. Therefore, $y_{n}$ converges, and hence

$$
\lim _{n \rightarrow \infty} y_{n}=\lim _{n \rightarrow \infty}\left(x_{n}+p_{n} x_{n-k}^{\alpha}\right)=\lim _{n \rightarrow \infty} x_{n}
$$

since $\lim _{n \rightarrow \infty} p_{n}=0$. Now, we consider Case (I). Since $y_{n}$ is a positive increasing sequence, there exist $n_{2} \geq n_{1}^{*}$ and $d>0$ such that

$$
x_{n}>d \quad \text { for } \quad n \geq n_{2} .
$$


Substituting (4) into (3), we get

$$
\Delta\left(a_{n} \Delta y_{n}\right)+q_{n} d^{\beta}<0 \quad \text { for } \quad n \geq n_{2} .
$$

Summing (5) from $n_{2}$ to $n-1$, we obtain

$$
a_{n} \Delta y_{n}-a_{n_{2}} \Delta y_{n_{2}}+d^{\beta} \sum_{s=n_{2}}^{n-1} q_{s}<0 \quad \text { for } \quad n \geq n_{2} .
$$

But (2) implies that

$$
\sum_{n=n_{2}}^{\infty} q_{n}=\infty
$$

which together with (6) yields

$$
\lim _{n \rightarrow \infty} a_{n} \Delta y_{n}=-\infty,
$$

a contradiction due to the eventual positivity of $a_{n} \Delta y_{n}$.

Next, we consider Case (II). Define the sequence $\left\{v_{n}\right\}$ by

$$
v_{n}=\frac{a_{n} \Delta y_{n}}{y_{n}} \quad \text { for } \quad n \geq n_{1} .
$$

Then $v_{n}<0$ for $n \geq n_{1}$. Also, the decreasing nature of $a_{n} \Delta y_{n}$ implies that

$$
\Delta y_{s} \leq \frac{a_{n}}{a_{s}} \Delta y_{n} \quad \text { for } \quad s \geq n \geq n_{1} .
$$

Summing (8) from $n$ to $r-1 \geq n$, we obtain

$$
y_{r}-y_{n} \leq a_{n} \Delta y_{n}\left(\sum_{s=n}^{r-1} \frac{1}{a_{s}}\right),
$$

which, by letting $r \rightarrow \infty$, leads to

$$
\frac{a_{n} \Delta y_{n}}{y_{n}} A_{n} \geq-1 \quad \text { for } \quad n \geq n_{1},
$$

i.e.,

$$
v_{n} A_{n} \geq-1 \quad \text { for } \quad n \geq n_{1} .
$$

On the other hand, we find from (9) that

$$
\Delta\left(\frac{y_{n}}{A_{n}}\right)=\frac{A_{n} \Delta y_{n}-y_{n} \Delta A_{n}}{A_{n} A_{n+1}}=\frac{A_{n} \Delta y_{n}+\frac{y_{n}}{a_{n}}}{A_{n} A_{n+1}} \geq 0,
$$

for $n \geq n_{1}$, and thus

$$
\frac{y_{n}}{A_{n}} \geq \frac{y_{n-k}}{A_{n-k}} \quad \text { for } \quad n \geq n_{1}+k .
$$

Now,

$$
x_{n}=y_{n}-p_{n} x_{n-k}^{\alpha} \geq y_{n}-p_{n} y_{n-k}^{\alpha} \quad \text { for } \quad n \geq n_{1}+k,
$$


and using (11), we obtain

$$
x_{n} \geq y_{n}-p_{n} \frac{A_{n-k}^{\alpha}}{A_{n}^{\alpha}} y_{n}^{\alpha}=\left(1-p_{n} \frac{A_{n-k}^{\alpha}}{A_{n}^{\alpha}} y_{n}^{\alpha-1}\right) y_{n} .
$$

Since $y_{n} / A_{n}$ is positive and increasing, we get

$$
\frac{y_{n}}{A_{n}} \geq \frac{y_{n_{1}}}{A_{n_{1}}}=: \gamma>0 \quad \text { for } \quad n \geq n_{1}
$$

Since $\left\{A_{n}\right\}$ is positive and converging to zero, there exists $n_{3} \geq n_{1}+k$ such that

$$
0<A_{n}^{\nu} \leq \gamma \quad \text { for all } \quad n \geq n_{3} .
$$

Hence, by (13) and (14),

$$
y_{n} \geq A_{n}^{1+\nu} \quad \text { for } \quad n \geq n_{3} .
$$

Using (15) in (12), we get

$$
x_{n} \geq\left(1-p_{n} \frac{A_{n-k}^{\alpha}}{A_{n}^{\alpha}} A_{n}^{(1+\nu)(\alpha-1)}\right) y_{n}=P_{n} y_{n} \quad \text { for } \quad n \geq n_{3} .
$$

By (16), from (3), we have

$$
\begin{aligned}
\Delta\left(a_{n} \Delta y_{n}\right) & =-q_{n} x_{n+1-m}^{\beta} \\
& \leq-q_{n} P_{n+1-m}^{\beta} y_{n+1-m}^{\beta} \\
& \leq-q_{n} P_{n+1-m}^{\beta} y_{n+1}^{\beta} \quad \text { for } \quad n \geq n_{3},
\end{aligned}
$$

where we also used the decreasing nature of $y_{n}$ in the last estimate. Now (17), in view of (15), leads to

$$
\begin{aligned}
\Delta\left(a_{n} \Delta y_{n}\right) & \leq-q_{n} A_{n+1}^{(1+\nu)(\beta-1)} P_{n+1-m}^{\beta} y_{n+1} \\
& =-Q_{n} y_{n+1} \quad \text { for } \quad n \geq n_{3} .
\end{aligned}
$$

Taking the difference of both sides of (7) and using the decreasing nature of $a_{n} \Delta y_{n}$, we get

$$
\begin{aligned}
\Delta v_{n} & =\frac{y_{n} \Delta\left(a_{n} \Delta y_{n}\right)-a_{n}\left(\Delta y_{n}\right)^{2}}{y_{n} y_{n+1}} \\
& =\frac{\Delta\left(a_{n} \Delta y_{n}\right)}{y_{n+1}}-\frac{y_{n}}{a_{n} y_{n+1}} v_{n}^{2} \\
& \leq \frac{\Delta\left(a_{n} \Delta y_{n}\right)}{y_{n+1}}-\frac{v_{n}^{2}}{a_{n}} \quad \text { for } \quad n \geq n_{3},
\end{aligned}
$$

where we have used again the decreasing nature of $y_{n}$. Combining (19) and (18), we have

$$
\Delta v_{n} \leq-Q_{n}-\frac{v_{n}^{2}}{a_{n}} \quad \text { for } \quad n \geq n_{3}
$$


Using (20), we get

$$
\begin{aligned}
\Delta\left(A_{n} v_{n}\right) & =v_{n} \Delta A_{n}+A_{n+1} \Delta v_{n} \\
& =-\frac{v_{n}}{a_{n}}+A_{n+1} \Delta v_{n} \\
& \leq-\frac{v_{n}}{a_{n}}-A_{n+1} Q_{n}-\frac{A_{n+1} v_{n}^{2}}{a_{n}} \\
& \leq-A_{n+1} Q_{n}+\frac{1}{4 a_{n} A_{n+1}},
\end{aligned}
$$

and summing this resulting inequality from $n_{3}$ to $n$ and using (10) yields

$$
\begin{aligned}
\sum_{s=n_{3}}^{n}\left[Q_{s} A_{s+1}-\frac{1}{4 a_{s} A_{s+1}}\right] & \leq A_{n_{3}} v_{n_{3}}-A_{n+1} v_{n+1} \\
& \leq 1+A_{n_{3}} v_{n_{3}}<\infty \text { for } n \geq n_{3},
\end{aligned}
$$

contradicting (2). This completes the proof.

When $\beta=1$, we have the following immediate corollary from Theorem 2.1 .

Corollary 2.1. Let $\beta=1$. Assume $\left(H_{1}\right)-\left(H_{3}\right)$. If

$$
\limsup _{n \rightarrow \infty} \sum_{s=n_{0}^{*}}^{n}\left[q_{s} P_{s+1-m} A_{s+1}-\frac{1}{4 a_{s} A_{s+1}}\right]=\infty,
$$

then (1) is oscillatory.

Next, we establish an oscillation result when $0<\beta<1$.

Theorem 2.2. Let $0<\beta<1$. Assume $\left(H_{1}\right)-\left(H_{3}\right)$. If

(22) $\limsup _{n \rightarrow \infty} \sum_{s=n_{0}^{*}}^{n}\left[L q_{s} P_{s+1-m}^{\beta} A_{s+1}-\frac{1}{4 a_{s} A_{s+1}}\right]=\infty$ for some $L>0$,

then (1) is oscillatory.

Proof. Let $x_{n}$ be a nonoscillatory solution of (1), say $x_{n}>0, x_{n+1-m}>0$, $x_{n-k}>0$, and $y_{n}>0$ for $n \geq n_{1}$ for some $n_{1} \geq n_{0}^{*}$. Proceeding as in the proof of Theorem 2.1, we obtain the two cases (I) $\Delta y_{n}>0$ or (II) $\Delta y_{n}<0$ for $n \geq n_{1}$. Next, we consider only Case (II) as Case (I) can be treated similarly as in the proof of Theorem 2.1. Recall that $y_{n}$ is positive and decreasing with $\lim _{n \rightarrow \infty} y_{n}=\lim _{n \rightarrow \infty} x_{n}$. Then we have either $\lim _{n \rightarrow \infty} y_{n}=d_{1}>0$ or $\lim _{n \rightarrow \infty} y_{n}=0$. The first case implies that $\lim _{n \rightarrow \infty} x_{n}=d_{1}$. Thus, there exist $d_{2}>0$ and $n_{1}^{*} \in \mathbb{N}$ such that $x_{n} \geq d_{2}$ for all $n \geq n_{1}^{*}$. Hence we can obtain a contradiction similarly as in Case (I). The other case implies that for $K:=L^{1 /(\beta-1)}>0$, there exists $n_{2}^{*} \in \mathbb{N}$ such that

$$
0<y_{n}<K \quad \text { for all } n \geq n_{2}^{*} \text {. }
$$


Now proceeding as in the proof of Theorem 2.1, we obtain (17), which with (23) yields

$$
\begin{aligned}
0 & \geq \Delta\left(a_{n} \Delta y_{n}\right)+q_{n} P_{n+1-m}^{\beta} y_{n+1}^{\beta} \\
& =\Delta\left(a_{n} \Delta y_{n}\right)+\frac{q_{n} P_{n+1-m}^{\beta} y_{n+1}}{y_{n+1}^{1-\beta}} \\
& \geq \Delta\left(a_{n} \Delta y_{n}\right)+\frac{q_{n} P_{n+1-m}^{\beta} y_{n+1}}{K^{1-\beta}} \\
& =\Delta\left(a_{n} \Delta y_{n}\right)+L q_{n} P_{n+1-m}^{\beta} y_{n+1} \quad \text { for } n \geq n_{3},
\end{aligned}
$$

with some $n_{3} \geq n_{2}^{*}$. The remainder of the proof is similar to that of Theorem 2.1 and hence is omitted.

\section{EXAmples AND REMARKS}

First, we give two examples for the case $\beta>1$.

Example 3.1. Consider the second-order equation

$$
\Delta\left(n(n+1) \Delta\left(x_{n}+\frac{x_{n-k}^{\alpha}}{n^{2}}\right)\right)+(n+1)^{6} x_{n+1-m}^{\frac{5}{3}}=0, \quad n \in \mathbb{N} .
$$

Here, $0<\alpha<1$ is a ratio of positive odd integers, $\beta=5 / 3$, the delays are $k \in \mathbb{N}$ and $m \in \mathbb{N}_{0}$, and

$$
a_{n}=n(n+1), \quad p_{n}=\frac{1}{n^{2}}, \quad \text { and } \quad q_{n}=(n+1)^{6} .
$$

We let $\nu=1$. It is easy to see that $\left(\mathrm{H}_{2}\right)$ holds. Also,

$$
A_{n}=\frac{1}{n} \quad \text { and } \quad P_{n}=1-\frac{1}{n^{\alpha}(n-k)^{\alpha}} .
$$

Moreover,

$$
Q_{n} A_{n+1}-\frac{1}{4 a_{n} A_{n+1}}=(n+1)^{\frac{11}{3}}\left[1-\frac{1}{(n+1-m)^{\alpha}(n+1-m-k)^{\alpha}}\right]^{\frac{5}{3}}-\frac{1}{4 n} .
$$

Thus,

$$
\lim _{n \rightarrow \infty}\left(Q_{n} A_{n+1}-\frac{1}{4 a_{n} A_{n+1}}\right)=\infty .
$$

Therefore, (2) of Theorem 2.1 is satisfied, and hence (24) is oscillatory.

Example 3.2. Consider the second-order equation

$$
\Delta\left(n(n+1) \Delta\left(x_{n}+\frac{x_{n-k}^{\alpha}}{n^{2}}\right)\right)+(n+1)^{2} x_{n+1-m}^{\frac{5}{3}}=0, \quad n \in \mathbb{N} .
$$

Here, all data are the same as in Example 3.1 except

$$
q_{n}=(n+1)^{2},
$$


and therefore

$$
Q_{n} A_{n+1}=\frac{1}{(n+1)^{\frac{1}{3}}}\left[1-\frac{1}{(n+1-m)^{\alpha}(n+1-m-k)^{\alpha}}\right]^{\frac{5}{3}} \geq \frac{1}{n} \cdot \frac{1}{2},
$$

for $n \geq N$ with some $N \in \mathbb{N}$, and thus

$$
\begin{aligned}
\sum_{n=N}^{M}\left(Q_{n} A_{n+1}-\frac{1}{4 a_{n} A_{n+1}}\right) & =\sum_{n=N}^{M}\left(Q_{n} A_{n+1}-\frac{1}{4 n}\right) \\
& \geq \sum_{n=N}^{M} \frac{1}{4 n} \rightarrow \infty \text { as } M \rightarrow \infty .
\end{aligned}
$$

Hence, (2) of Theorem 2.1 is satisfied, and thus (25) is oscillatory.

Next, we give an example in the case $\beta=1$.

Example 3.3. Consider the second-order equation

$$
\Delta\left(n(n+1) \Delta\left(x_{n}+\sqrt[3]{\frac{(n-k) x_{n-k}}{8 n^{4}}}\right)\right)+\frac{n+1}{n} x_{n+1-m}=0, \quad n \in \mathbb{N} .
$$

Here, $\alpha=1 / 3, \beta=1$, the delays are $k \in \mathbb{N}$ and $m \in \mathbb{N}_{0}$, and

$$
a_{n}=n(n+1), \quad p_{n}=\sqrt[3]{\frac{n-k}{8 n^{4}}} \quad \text { and } \quad q_{n}=\frac{n+1}{n} .
$$

We let $\nu=1 / 2$. It is easy to see that $\left(\mathrm{H}_{2}\right)$ holds. Also,

$$
A_{n}=\frac{1}{n} \quad \text { and } \quad P_{n}=\frac{1}{2} \text {. }
$$

Moreover,

$$
q_{n} P_{n+1-m} A_{n+1}-\frac{1}{4 a_{n} A_{n+1}}=\frac{q_{n}}{2(n+1)}-\frac{1}{4 n}=\frac{1}{4 n} .
$$

Therefore, (21) of Corollary 2.1 is satisfied, and hence (26) is oscillatory.

Finally, we present an example in the case $0<\beta<1$.

Example 3.4. Consider the second-order equation

$$
\Delta \frac{\Delta\left(x_{n}+4^{(\alpha-1)(n-1)-(k \alpha+1) / 2} x_{n-k}^{\alpha}\right)}{2^{n}}+n 8^{n} x_{n+1-m}^{\beta}=0, \quad n \in \mathbb{N} .
$$

Here, $0<\alpha<1$ and $0<\beta<1$ are ratios of positive odd integers, the delays are $k \in \mathbb{N}$ and $m \in \mathbb{N}_{0}$, and

$$
a_{n}=\frac{1}{2^{n}}, \quad p_{n}=4^{(\alpha-1)(n-1)-(k \alpha+1) / 2} \quad \text { and } \quad q_{n}=n 8^{n} .
$$

We let $\nu=1$. It is easy to see that $\left(\mathrm{H}_{2}\right)$ holds. Also,

$$
A_{n}=\frac{1}{2^{n-1}} \quad \text { and } \quad P_{n}=\frac{1}{2} \text {. }
$$


Moreover,

$$
L q_{n} P_{n+1-m}^{\beta} A_{n+1}-\frac{1}{4 a_{n} A_{n+1}}=\operatorname{Ln} 2^{2 n-\beta}-2^{2 n-2},
$$

which tends to infinity for any constant $L>0$. Therefore, (22) of Theorem 2.2 is satisfied, and hence (27) is oscillatory.

Remark 3.1. The results of this paper are presented in a form that makes it easy to study extensions to higher-order equations. It would also be of interest to use the approach here to study (1) with $\alpha>1$, i.e., (1) with superlinear neutral term.

Remark 3.2. Another possibility for extension of the presented results would be to consider the time-scales $[5,8]$ analogue of (1).

\section{REFERENCES}

[1] R. P. Agarwal, Difference equations and inequalities: Theory, methods, and applications, Monographs and Textbooks in Pure and Applied Mathematics, Marcel Dekker, Inc., New York, 2nd Ed., 2000.

[2] R. P. Agarwal, M. Bohner, S. R. Grace, D. O'Regan, Discrete oscillation theory, Hindawi Publishing Corporation, New York, 2005.

[3] R. P. Agarwal, M. Bohner, T. Li, C. Zhang, Oscillation of second-order differential equations with a sublinear neutral term, Carpathian J. Math., 30 (1) (2014), 1-6.

[4] R. P. Agarwal, S. R. Grace, D. O'Regan, Oscillation theory for difference and functional differential equations, Kluwer Academic Publishers, Dordrecht, 2000.

[5] M. Bohner, S. G. Georgiev, Multivariable dynamic calculus on time scales, Springer, Cham, 2016.

[6] M. Bohner, S. R. Grace, I. Jadlovská, Oscillation criteria for second-order neutral delay differential equations, Electron. J. Qual. Theory Differ. Equ., 2017, No. 60, $1-12$.

[7] M. Bohner, T. Li, Oscillation of second-order p-Laplace dynamic equations with a nonpositive neutral coefficient, Appl. Math. Lett., 37 (2014), 72-76.

[8] M. Bohner, A. Peterson, Dynamic equations on time scales: An introduction with applications, Birkhäuser Boston, Inc., Boston, MA, 2001.

[9] C. Dharuman, J. R. Graef, E. Thandapani, K. S. Vidhyaa, Oscillation of second order difference equation with a sub-linear neutral term, J. Math. Appl., 40 (2017), 59-67.

[10] H. A. El-Morshedy, Oscillation and nonoscillation criteria for half-linear second order difference equations, Dynam. Systems Appl., 15 (3-4) (2006), 429-450. 
[11] H. A. El-Morshedy, New oscillation criteria for second order linear difference equations with positive and negative coefficients, Comput. Math. Appl., 58 (10) (2009), 1988-1997.

[12] H. A. El-Morshedy, S. R. Grace, Comparison theorems for second order nonlinear difference equations, J. Math. Anal. Appl., 306 (1) (2005), 106-121.

[13] S. R. Grace, R. P. Agarwal, M. Bohner,D. O'Regan, Oscillation of second-order strongly superlinear and strongly sublinear dynamic equations, Commun. Nonlinear Sci. Numer. Simul., 14 (8) (2009), 3463-3471.

[14] S. R. Grace, M. Bohner, R. P. Agarwal, On the oscillation of second-order half-linear dynamic equations, J. Difference Equ. Appl., 15 (5) (2009), 451-460.

[15] S. R. Grace, H. A. El-Morshedy, Oscillation criteria of comparison type for second order difference equations, J. Appl. Anal., 6 (1) (2000), 87-103.

[16] S. R. Grace, J. R. Graef, Oscillatory behavior of second order nonlinear differential equations with a sublinear neutral term, Math. Model. Anal., 23 (2) (2018), 217-226.

[17] J. R. Graef, S. R. Grace, E. Tunç, Oscillatory behavior of even-order nonlinear differential equations with a sublinear neutral term, Opuscula Math., 39 (1) (2019), $39-47$.

[18] W.-T. Li, S. H. Saker, Oscillation of second-order sublinear neutral delay difference equations, Appl. Math. Comput., 146 (2-3) (2003), 543-551.

[19] S. H. Saker, Oscillation of superlinear and sublinear neutral delay dynamic equations, Commun. Appl. Anal., 12 (2) (2008), 173-187.

[20] S. Selvarangam, M. Madhan, E. Thandapani, Oscillation theorems for second order nonlinear neutral type difference equations with positive and negative coefficients, Rom. J. Math. Comput. Sci., 7 (1) (2017), 1-10.

[21] S. Selvarangam, E. Thandapani, S. Pinelas, Oscillation theorems for second order nonlinear neutral difference equations, J. Inequal. Appl. 2014, 2014:417.

[22] A. K. Sethi, Oscillation of second order sublinear neutral delay dynamic equations via Riccati transformation, J. Appl. Math. Inform., 36 (3-4) (2018), 213-229.

[23] A. K. Tripathy, A. K. Sethi, Oscillation of sublinear second order neutral differential equations via Riccati transformation, In: Differential and difference equations with applications, Springer Proc. Math. Stat., 230 (2018), 543-557.

[24] M. K. Yildiz, H. Öğ̈̈nmez, Oscillation results of higher order nonlinear neutral delay difference equations with a nonlinear neutral term, Hacet. J. Math. Stat., 43 (5) (2014), 809-814. 
MARTIN BOHNER

Department of Mathematics and Statistics

Missouri S\&T

ROLLA, MO 65409-0020

USA

E-mail address: bohner@mst.edu

Hassan A. El-Morshedy

Department of Mathematics

Faculty of Science

DAMIETTA University

New Damietta 34517

EGYPT

E-mail address: elmorshedy@yahoo.com

Said R. Grace

Department of Engineering Mathematics

FACUlTy OF EngINEERING

Cairo University

Orman, Giza 12000

EGYPT

E-mail address: saidgrace@yahoo.com

\section{ILGIN SAĞER}

Department of Mathematics and Computer Science University of Missouri-St. Louis

ST. Louis, MO 63121-4400

USA

E-mail address: sageri@umsl.edu 